\title{
Publisher's Note: Relaxation dynamics of ultracold bosons in a double-well potential: Thermalization and prethermalization in a nearly integrable model [Phys. Rev. A 92, 033607 (2015)]
}

\author{
Jayson G. Cosme and Oleksandr Fialko \\ (Received 6 November 2015; published 23 November 2015)
}

DOI: 10.1103/PhysRevA.92.059903

PACS number(s): 67.85.-d, 05.70.Ln, 05.45.Mt, 05.30.-d, 99.10.Fg

This paper was published online on 10 September 2015 with the omission of an author in the author list and of his byline information. Oleksandr Fialko should appear as the second author, and his byline affiliation should read as "Institute of Natural and Mathematical Sciences, Dodd-Walls Centre for Photonics and Quantum Technology, Centre for Theoretical Chemistry and Physics, Massey University Auckland, Private Bag 102904, North Shore, Auckland 0745, New Zealand.” In addition, there are errors in Eqs. (8) and (22). Equation (8) should read as

$$
|k\rangle=\sum_{\{n\}} C_{n}^{k}|n\rangle .
$$

Equation (22) should read as

$$
S_{\mathrm{vN}}(t)=-\operatorname{tr}\left[\hat{\rho}_{s} \log \hat{\rho}_{s}\right]=-\sum_{m=0}^{N} \lambda_{m}(t) \log \lambda_{m}(t) .
$$

The equations have been corrected as of 23 October 2015. The equations are incorrect in the printed version of the journal. 